\title{
UCLA RADIOCARBON DATES XI
}

\author{
RAINER BERGER
}

Institute of Geophysics, Departments of Geography and Anthropology

Interdisciplinary Archaeology Graduate Program

University of California, Los Angeles, California 90024

\section{REINER PROTSCH}

Anthropologisches Institut, Johann-Wolfgang-Goethe Universität Frankfurt am Main, FGR

\section{INTRODUCTION}

The radiocarbon dates reported in this list are almost all based on collagen measurements from human archaeologic bone material. This collagen was isolated according to the methods developed by Berger, Horney and Libby (1964), Ho, Marcus and Berger (1969), Longin (1971) and Protsch (1973). All samples were counted as $\mathrm{CO}_{2}$ gas at close to 1 atm in a $7.5 \mathrm{~L}$ proportional counter with three energy channels. The ${ }^{14} \mathrm{C}$ dates reported are calculated with the Libby half-life of $5568 \pm 30$ years as required by convention. ${ }^{13} \mathrm{C}$ measurements were used to normalize to $-25 \%$ in the standard manner. The biospheric standard is $95 \%$ the count rate of NBS oxalic acid for radiocarbon laboratories. Background is based on $\mathrm{CO}_{2}$ obtained from marble. The accuracy of counter operation is checked against historically dated wood from the funerary boat of Sesostris III, 1872 -8 BC (Hayes, 1962). The ${ }^{14} \mathrm{C}$ ages are not tree-ring calibrated.

\section{ACKNOWLEDGMENTS}

We are indebted to the National Science Foundation and the Deutsche Forschungsgemeinschaft for support and numerous donors for sample materials. Suzanne DeAtley provided invaluable laboratory expertise. I R Kaplan and D Winter analyzed ${ }^{13} \mathrm{C} /{ }^{12} \mathrm{C}$ ratios.

\section{United States}

\section{California}

UCLA-1008A. Abalone Cove

Surface find from Gabrielino Indian site on second terrace at Abalone Cove, Palos Verdes, Los Angeles Co $\left(33^{\circ} 44^{\prime} \mathrm{N}, 118^{\circ} 23^{\prime} \mathrm{W}\right)$. Subm by M Reiter, Geol Dept, Los Angeles Harbor Coll.

\section{UCLA-1008B. Abalone Cove}

Surface find from site near location of UCLA-1008A. Comment (MR): dates show occupation of same general area at different times by Gabrielino Indians.

\section{UCLA-1088. Drake site}

Fragmentary bones of female skeleton, heavily coated with caliche, from $1.5 \mathrm{~m}$ depth in reddish midden layer under $1 \mathrm{~m}$ of black midden at 
Drake site off Toro Canyon Rd, Santa Barbara Co $\left(34^{\circ} 24^{\prime}\right.$ N, $\left.119^{\circ} 35^{\prime} \mathrm{W}\right)$. Coll July 1966 by P C Orr, Santa Barbara Mus Nat Hist. Comment (PCO): burial was exposed by ditch digging equipment and, due to caliche coating, thought to be of considerable age. Indeed, date fits Oak Grove culture.

\section{UCLA-1092. San Simeon}

$3180 \pm 600$

Fragmentary bones from Little Pico Creek site near San Simeon, San Louis Obispo Co $\left(35^{\circ} 39^{\prime} \mathrm{N}, 121^{\circ} 11^{\prime} \mathrm{W}\right)$. Coll Sept 1965 by D Abrams from coastal site with shell midden and artifacts such as sweat stick bone awls. Comment (DA): oldest date for site since sample originated from location just above bedrock.

UCLA-1411. Medea Creek

$\mathbf{3 5 0} \pm \mathbf{5 0}$

Fragmentary bones from Medea Creek site, 4-Ven-243, Ventura Co, burial no. $73\left(34^{\circ} 16^{\prime} \mathrm{N}, 118^{\circ} 40^{\prime} \mathrm{W}\right)$. Subm by UCLA archaeology field training class.

\section{UCLA-1425A. Stanford Man II}

$4400 \pm 270$

Cranial bone from Stanford Man II skeletal remains $\left(37^{\circ} 26^{\prime} \mathrm{N}, 122^{\circ}\right.$ $12^{\prime} \mathrm{W}$ ). Nitrogen content of bones was $2.0 \%$ and fluorine content $0.27 \%$. Coll and subm June 1966 by B Gerow, Stanford Univ through C Rozaire, Los Angeles Co Mus Nat Hist.

UCLA-1425B. Stanford Man II

$4350 \pm 130$

Right femur from Stanford Man II colln.

UCLA-1861. Stanford Man I

$5130 \pm 70$

Cranial bone from Stanford Man I found $100 \mathrm{~m}$ downstream from Stanford Man II locus and ca $\mathrm{lm}$ lower in deposit. Bone nitrogen content was $2.1 \%$ indicating ca $5 \%$ collagen present in bone. Comment (RB): originally Stanford Man was thought to represent Early Man in the Americas, which these dates refute.

UCLA-1430. Los Angeles Man

Cranial bone from skull fragment found Jan 1936 during WPA Proj C-642 N of Baldwin Hills, City of Los Angeles $\left(34^{\circ} \mathrm{N}, 118^{\circ} \mathrm{W}\right)$. Date based on bone with $0.32 \%$ nitrogen and $0.11 \%$ fluorine content from which purified amino acid fraction was isolated similar to procedure of Ho, Marcus and Berger (1969) to avoid contamination by petroleum residues found in area. Comment (RB): since sample was in stratigraphic relation to mammoth bones in same river bed and is very dark brown, we thought it might be of Pleistocene age (Berger, 1975; Taylor et al, 1985). To settle discrepancies in dating, an independent AMS date is desirable.

\section{UCLA-1431. Chiquito Springs}

$780 \pm 70$

Fragment of humerus coll by L Lattimer from Chiquito Springs, Orange Co $\left(33^{\circ} 38^{\prime} \mathrm{N}, 117^{\circ} 28^{\prime} \mathrm{W}\right)$ during archaeologic survey of this inland location. 
Bone fragments from site Ora-58 in Costa Mesa, Orange Co $\left(33^{\circ} 39^{\prime}\right.$ $\left.\mathrm{N}, 117^{\circ} 56^{\prime} \mathrm{W}\right)$. Subm by K Dixon, California State Univ, Long Beach. Comment $(\mathrm{KD})$ : date fits well with expected age for site.

\section{UCLA-1498A. Del Mar}

$3300 \pm 100$

Bone fragments from Del Mar W-20, S of Interstate 5 at Del Mar, San Diego Co $\left(32^{\circ} 57^{\prime} \mathrm{N}, 117^{\circ} 15^{\prime} \mathrm{W}\right)$ at $2.4 \mathrm{~m}$ depth. Charcoal from same level dates to $3400 \pm 60$ (UCLA-1498B). Coll by E L Davies. Comment (ELD): sample originates from lower half of flexed burial without grave goods typical of La Jollan stage. Earliest habitation level at W-20.

UCLA-1499. Torrey Pines Mesa

$4100 \pm 250$

Bone from site W-340, flexed burial, on Torrey Pines Mesa, San Diego $\left(32^{\circ} 54^{\prime} \mathrm{N}, 117^{\circ} 14^{\prime} \mathrm{W}\right) 15 \mathrm{~cm}$ below surface. Coll Sept 1968 by E L Davies. Comment (ELD): similar age range as UCLA-1498A, above, due to comparable archaeology.

\section{UCLA-1623. Tranquillity Man}

$2550 \pm 60$

Fragmentary bone material from burials 3 and 4 subm by R F Heizer and A B Elsasser, Univ California, Berkeley. Suspected Early Man site since burials and Late Pleistocene fauna are in close proximity at this location $\left(36^{\circ} 39^{\prime} \mathrm{N}, 120^{\circ} 17^{\prime} \mathrm{W}\right)$. Comment (RB): burials intrude into Pleistocene deposits as discussed by Berger et al (1971).

UCLA-1631. Ventura Oak Grove

$1030 \pm 80$

Burial 1 of Site Ven-150, Ventura $\left(34^{\circ} 16^{\prime} \mathrm{N}, 119^{\circ} 18^{\prime} \mathrm{W}\right)$ at $50 \mathrm{~cm}$ depth. Coll June 1968 by R Browne. Comment (RB): age estimate fits archaeologic context.

UCLA-1641. Cemetery LAn-243

$470 \pm 60$

Bone fragments from Site LAn-243, Los Angeles Co $\left(34^{\circ} \mathrm{N}, 118^{\circ} 15^{\prime}\right.$ W). Six separate bones were dated with nitrogen content ranging from 2.26 to $3.42 \%$ and fluorine 0.32 to $1.22 \%$. Coll by UCLA field class to study environmental differences at same site.

\section{UCLA-1686A. Diablo Canyon}

$9320 \pm 140$

Burial 20, Site D-1, Diablo Canyon, San Luis Obispo Co $\left(35^{\circ} 16^{\prime} \mathrm{N}\right.$, $120^{\circ} 40^{\prime} \mathrm{W}$ ) at 320 to $330 \mathrm{~cm}$. Date based on skull bone fragments containing $1.06 \%$ nitrogen. Subm June 1971 by R Greenwood.

UCLA-1686B. Diablo Canyon

$930 \pm 80$

Right femur from Burial 44, Site D-1, same location as UCLA-1686A from $120 \mathrm{~cm}$ depth. Subm by R Greenwood. Comment (RG): this site has one of longest continuous habitations found on US W coast. 
UCLA-1694. Vasquez Rocks

Bone from LAn-361 (Asher No.1) at Vasquez Rocks, Los Angeles Co $\left(34^{\circ} 35^{\prime} \mathrm{N}, 118^{\circ} 7^{\prime} \mathrm{N}\right)$. Subm by C Rozaire. Comment (CR): this date establishes difference between cremation and interment levels at upper Santa Clara River Valley in mountains $\mathrm{N}$ of Los Angeles.

UCLA-1712. Truckhaven

Bone fragments from burial near Salton Sea, San Diego Co $\left(33^{\circ} 27^{\prime} \mathrm{N}\right.$, $\left.116^{\circ} 5^{\prime} \mathrm{W}\right)$. Coll and subm by W Childers, Imperial Valley Coll, El Centro. Comment (WC): area around burial contained stone tools similar to those found in Lake Manix, San Bernardino Co basin and thought to be possibly of Early Man age. Collagen date is much more recent, perhaps due to intrusive burial.

UCLA-1771 B. Castaic site

$2040 \pm 350$

Occipital and portions of parietal and temporal bones from Castaic site LAn-324, Los Angeles Co (34 $\left.24^{\prime} \mathrm{N}, 118^{\circ} 34^{\prime} \mathrm{W}\right)$ at mouth of Elderberry Canyon. Coll June 1971 by $\mathrm{H}$ E Hanks and C A Singer in area of house rings, cairns, and roasting pits. Comment (CAS): sample provides absolute date for upper area of site.

\section{Mostin site series}

Several important Early Man sites are located at Clear Lake, $160 \mathrm{~km} \mathrm{~N}$ of San Francisco. Borax Lake site was excavated by Meighan and Haynes (1970) and thought to be ca 10,000 yr old. The Mostin site, near Kelseyville $\left(38^{\circ} 58^{\prime} \mathrm{N}, 122^{\circ} 50^{\prime} \mathrm{W}\right)$ has become one of the oldest examples of obsidian use in the Americas. Samples listed below were discussed by Ericson and Berger (1974).

UCLA-1795A. Mostin site

$10,260 \pm 340$ Co.

Right femur and left fibula from Burial 4, Mostin site, SDA-66, Lake

UCLA-1795B. Mostin site

$9040 \pm 200$

Right and left femur fragments from Burial 1. Nitrogen content of bones was $0.38 \%$.

UCLA-1795C. Mostin site

$\mathbf{7 7 5 0} \pm \mathbf{4 0 0}$

Left ulna, femur, and humerus from Burial 9.

General Comment (RB): since Mostin site is being exposed by erosion of banks of Kelsey Creek, numerous burials have been lost during annual rainy seasons of last several years. More detailed results for Mostin site are discussed in Kaufman (1980).

UCLA-1885. Van Norman Reservoir

$600 \pm 50$

Ribs from Burial 1 at Site No. 6, Van Norman Reservoir, 4-LAn-629, Los Angeles (34 $\left.17^{\prime} \mathrm{N}, 118^{\circ} 29^{\prime} \mathrm{W}\right)$. Subm by C King. Comment (CK): this 
burial of tight-flexed male, 20-25 yr old, dates Phase 1 of Late Horizon in central California sequence.

UCLA-1886. Malibu

$1250 \pm 60$

Bone from Burials 35 and 36, Malibu site, LAn-264, excavated Spring 1973 by $\mathrm{C}$ King $\left(34^{\circ} 3^{\prime} \mathrm{N}, 118^{\circ} 45^{\prime} \mathrm{W}\right)$. Nitrogen content of bones was $3.57 \%$. Comment (CK): rib samples from two stratigraphically contemporary burials of Chumash Indians.

UCLA-1890. San Luis Obispo

$1460 \pm 60$

Burial 8 of Site 4-SLO-406 excavated by C King and R Gibson $\left(35^{\circ} 16^{\prime}\right.$ $\mathrm{N}, 120^{\circ} 40^{\prime} \mathrm{W}$ ). Comment (CK): date fits expected age.

UCLA-1891A. Marin

$3270 \pm 70$

Burial 4 at Site-152, Marin Co $\left(37^{\circ} 54^{\prime} \mathrm{N}, 122^{\circ} 34^{\prime} \mathrm{W}\right)$ excavated by W Clewlow. Date based on left femur of female adult found at $90 \mathrm{~cm}$ depth.

UCLA-1891B. Marin

$3050 \pm 130$

Burial 5 at same site as UCLA-1891A, right humerus from $90 \mathrm{~cm}$ depth. Comment (WC): both burials were assoc with obsidian and fit into Middle Horizon of central California sequence.

UCLA-1899. Arlington Springs

$10,080 \pm 810$

Long bone fragment from Arlington Springs Early Man site, Santa Rosa I. $\left(34^{\circ} \mathrm{N}, 120^{\circ} 5^{\prime} \mathrm{W}\right)$. This site was previously dated by Olson and Broecker (1961) at 10,000 yr (L-650) using charcoal (Orr, 1968). Comment (RB): earliest human bone date on Channel Is. of California determined using UCLA $200 \mathrm{ml} \mathrm{CO}$ proportional counter. This date also shows that Channel Is. were indeed reached by water craft from mainland by $10,000 \mathrm{yr}$ ago.

UCLA-1924. Angeles Mesa

$10,500 \pm 2000$

Femur marked 3 from Angeles Mesa skeletons thought to be of Early Man age. To remove possible surface contamination, bone was extracted for 10 days continuously with a 1:1 ethyl ether/ethyl alcohol mixture in Soxhlet apparatus and then dried at $110^{\circ} \mathrm{C}$ for $24 \mathrm{hr}$. Subm by L Crum and G Kennedy, UCLA. Comment (LC \& GK): date confirms our age estimate.

UCLA-1933A. Tehachapi

$210 \pm 70$

Bone fragments from Site AVC-7 near Tehachapi (35 ${ }^{\circ} 8^{\prime} \mathrm{N}, 118^{\circ} 27^{\prime}$ W). Nitrogen content of bone was $2.94 \%$. Coll and subm by $\mathrm{E}$ Cole and $\mathrm{R}$ Robinson. Comment (EC): this salvage excavation of Shoshonian period site concentrated on understanding late Antelope Valley archaeology marked by more intense environmental exploitation and larger settlements.

\section{UCLA-1973. Santa Rosa Island}

$7650 \pm 580$

$\delta^{13} C=-22.7 \%$ o

Long bone fragment from burial eroding at mouth of Lobo Canyon, Santa Rosa I. $\left(34^{\circ} \mathrm{N}, 120^{\circ} 5^{\prime} \mathrm{W}\right)$. Coll by C Haupt, B, J and M Woolley and 
$\mathrm{R}$ Berger. Nitrogen content of bone was $0.73 \%$. Comment $(\mathrm{RB}):{ }^{13} \mathrm{C}$ ratio indicates little dependence on marine resources.

UCLA-2522. Glenwood

$$
\begin{array}{r}
2660 \pm 220 \\
\delta^{13} C=-26.14 \%
\end{array}
$$

Bone fragments from Glenwood, San Rafael Hills, Los Angeles Co $\left(34^{\circ} 13^{\prime} \mathrm{N}, 119^{\circ} 2^{\prime} \mathrm{W}\right)$, Site no. LAn-807. Coll and subm by M Boxt. Comment $(\mathrm{MB}):{ }^{13} \mathrm{C}$ ratio suggests no dependence on cereals at all.

\section{Illinois}

\section{Illinois Mounds Series I}

Bone material was excavated in Calhoun, Green and Pike Counties mounds by J Tainter, Northwestern Univ, Evansville, Illinois along Mississippi R, N of St Louis.

\section{UCLA-1919A. Schild Mounds}

$1160 \pm 50$

Ribs from Grave 5, Mound 9, Schild Mound group, Greene Co along Mississippi R ( $\left.39^{\circ} 32^{\prime} \mathrm{N}, 91^{\circ} \mathrm{W}\right)$. Interment was in log-roofed tomb (Perino, 1973).

UCLA-1919B. Schild Mounds

$$
1260 \pm 40
$$

Ribs from Burial 7, Mound 9.

UCLA-1919C. Schild Mounds

$1130 \pm 50$

Ribs from Burial 34a, Mound 3 (Perino, 1973).

UCLA-1919D. Koster Mounds

$1310 \pm 50$

Ribs from Burial 17, Mound 5, Koster Mound group, Greene Co.

UCLA-1919E. Koster Mounds

$1340 \pm 70$

Ribs from Burial 18, Mound 5.

UCLA-1919F. Ledders Mounds

$950 \pm 30$

Ribs from Burial 76, Mound 1, Ledders Mound group, Calhoun Co. Assoc with red-slipped, shell-tempered Mississippian pottery.

UCLA-1919G. Ledders Mounds

$\mathbf{8 9 0} \pm \mathbf{5 0}$

Ribs from Burial 81, Mound 1. Similar pottery as UCLA-1919F.

UCLA-1919H. Ledders Mounds

$\mathbf{9 7 0} \pm \mathbf{5 0}$

Ribs from Burial 135, Mound 1. This burial was interred in common grave with 76 and 81 .

UCLA-1919I. Joe Gay Mounds

$$
\mathbf{8 3 0} \pm \mathbf{8 0}
$$

Charred bone from crematory in Joe Gay Mounds group which contained White Hall phase potsherds dating between AD 400 to 700 (Struever, 1968). 
Ribs from Burial 3, Mound 6, Homer Adams Mounds in Pike Co.

UCLA-1919K. Joe Gay Mounds

Ribs from Burial 1, 12, 14, and 22, Joe Gay Mounds, Pike Co.

General Comment (JT): UCLA-1919A-H fit expected age range. UCLA1919I seems too recent in light of pottery age. UCLA-1919J-K also appear to be too early, as Late Woodland ca AD 400 would be expected.

\section{Illinois Mounds series II}

Mound excavations at Helton site $\left(39^{\circ} 32^{\prime} \mathrm{N}, 91^{\circ} \mathrm{W}\right)$ were carried out by J Buikstra, Univ Chicago. All bone samples show no pathology and are discussed in detail in Conner (1985).

UCLA-1946A. Helton Mounds $1380 \pm 90$

Ribs from Helton Mound 22, Sample 22-32-1.

UCLA-1946B. Helton Mounds

$$
520 \pm 80
$$

Ribs from Helton Mound 22, Sample 22-33-1.

UCLA-1946C. Helton Mounds

Ribs from Helton Mound 21, Sample 22-7-1.

UCLA-1946D. Helton Mounds.

Ribs from Helton Mound 21, Sample 21-11-1.

UCLA-1946E. Helton Mounds

Ribs from Helton Mound 20, Sample 20-36-2.

UCLA-1946F. Helton Mounds

$1720 \pm 100$

Ribs from Helton Mound 20, Sample 20-36-7.

UCLA-1946G. Helton Mounds

$200 \pm 60$

Ribs from intrusive burial at Helton 20-24.

UCLA-1946H. Helton Mounds

$1280 \pm 60$

Ribs from Helton Mound 20, Sample 37.

UCLA-1946I. Helton Mounds

$940 \pm 70$

Ribs from off-mound burial, Helton Mound 20. Sample 64.

UCLA-1946J. Helton Mounds

$850 \pm 70$

Ribs from sub-floor burial, Helton Mound 20. Sample 14.

UCLA-1946K. Helton Mounds

$730 \pm 60$

Ribs from sub-floor burial, Helton Mound 20. Sample 27.

General Comment (RB): dates reported here have been rounded off to nearest decade as opposed to their original quote to archaeologic investigator due to changes in editorial policy. 
Iowa

UCLA-2105. Lewis Central School

Left femur from articulated burial in ossuary Site 13 PW 5, Lewis School site $\left(41^{\circ} 13^{\prime} \mathrm{N}, 95^{\circ} 48^{\prime} \mathrm{W}\right)$. Assoc with side-notched points. Coll Nov 1975 by J A Hotopp; subm R Shutler, Jr.

Nevada

UCLA-1459C. Lovelock Cave

$3380 \pm 60$

Right tibia from Lovelock Cave, NV-CH-18 $\left(40^{\circ} 10^{\prime} \mathrm{N}, 118^{\circ} 30^{\prime} \mathrm{W}\right)$ from excavation of R F Heizer and L Napton, Univ California, Berkeley. Specimen from Burial F-5 with 4.5\% nitrogen content and 0.11 fluorine.

New Mexico

UCLA-1432. Bishop's Cap Cavern

$5320 \pm 120$

Unpreserved, femur, see UCLA-1059 (Berger \& Libby, 1969) for earlier date. Coll 1929 by H Wylde, L Lytle and W Bliss; subm by C Rozaire. Field loc no. 1009. Comment (RB): cavern contained Pleistocene animal bones and human remains leading to suspicion that this is an Early Man site. Radiocarbon date disproves this supposition.

Utah

UCLA-1942. Moab Woman

$210 \pm 70$

Left humerus, copper-impregnated, $15 \mathrm{~m}$ below overburden near Moab $\left(38^{\circ} 35^{\prime} \mathrm{N}, 109^{\circ} 34^{\prime} \mathrm{W}\right)$. Subm by L Ottinger and J E Ericson, Univ California, Irvine. Comment (JEE): deep burial was thought to indicate substantial age, but direct date disproves this theory.

\section{Belize}

UCLA-1938C. Patchchacan

$760 \pm 110$

Bone from Burial 14-2 at Patchchacan (13 $\left.23^{\prime} \mathrm{N}, 88^{\circ} 23^{\prime} \mathrm{W}\right)$. Coll and subm by R Sydris, UCLA.

UCLA-1938D. Sartineja

Bone from Burials 1,2 and 3 at Sartineja $\left(18^{\circ} 23^{\prime} \mathrm{N}, 88^{\circ} 23^{\prime} \mathrm{W}\right)$. Coll and subm by $\mathbf{R}$ Sydris.

\section{Guatemala}

UCLA-1439. Colima

Colima shaft tomb $\left(19^{\circ} 14^{\prime} \mathrm{N}, 103^{\circ} 41^{\prime} \mathrm{W}\right)$. Coll and subm by $\mathrm{G}$ C Kennedy. Comment (RB): shells from same tomb previously dated to $2090 \pm 50$ (UCLA-1438) uncorrected. This implies re-use of tomb.

\section{Seibal series}

Several burials were examined by $\mathrm{G}$ Willey, Harvard Univ at Seibal $\left(16^{\circ}\right.$ $\left.35^{\prime} \mathrm{N}, 90^{\circ} 5^{\prime} \mathrm{W}\right)$. Details were pub by Berger et al (1974). 
UCLA-1640A. Seibal

Bone from Burial 12 below patio floor of Structure 31A.

UCLA-1640B. Seibal

Bone from Burial 13 inside Structure 26d.

UCLA-1640C. Seibal

$1420 \pm 100$

Burial 17 inside Structure A2.

UCLA-1640D. Seibal

$1490 \pm 320$

Bone from Burial 19 beneath platform in front of structure D3.

UCLA-1640F. Seibal

$1100 \pm 100$

Bone from mass burial No. 4 in Structure a-13.

General Comment (RB): this series narrows interval between beginning of lowland Maya occupation and Olmec centers on Gulf of Mexico, allowing for coexistence of quite different cultures in relatively close geographic proximity.

\section{Mexico}

UCLA-1651. Parranda

Bone from Burial a, Pit 3, Colima site A. Coll and subm by I Kelly.

UCLA-1773. Astahuacan Man

$10,300 \pm 600$

Bone fragments from Astahuacan Man found by G C O'Neill in Valley of Mexico assoc with bison $\left(19^{\circ} 18^{\prime} \mathrm{N}, 98^{\circ} 52^{\prime} \dot{W}\right)$. Sample had nitrogen content of $2.71 \%$ and $0.06 \%$ fluorine. Comment (RB): date shows contemporaneity of Early Man finds in US with Mexico ca 10,000 yr ago.

\section{Peru}

UCLA-2179. Peruvian Mummy

Harvard Univ Mummy no. 1 subm by E Trinkhaus and J E Ericson. Comment (JEE): date proves mummy to be of recent origin.

\section{Greece}

UCLA-1881. Hydria

$2450 \pm 100$

Bone fragments of 2-yr-old infant entombed in Greek hydria. Comment (RB): stylistic design of hydria is confirmed by ${ }^{14} \mathrm{C}$ date (Berger \& Protsch, 1975).

\section{Italy}

UCLA-1915. Italian Neolithic

$5020 \pm 60$

Bone from Neolithic tomb contents $\mathrm{N}$ of Naples $\left(40^{\circ} 50^{\prime} \mathrm{N}, 14^{\circ} 15^{\prime} \mathrm{W}\right)$. Subm by R Panza, Bari. 
UCLA-2148. Lagnano

Bone from Lagnano da Piede near Foggia $\left(41^{\circ} 28^{\prime} \mathrm{N}, 15^{\circ} 33^{\prime} \mathrm{E}\right)$ at 65 to $105 \mathrm{~cm}$ depth, sec 1, 2 and 3 assoc with pottery. Coll by S Skomal; subm by N Kidd and M Gimbutas, UCLA. Comment (MG): dates some of earliest pottery of Italy.

The Netherlands

UCLA-1856C. Haan

$1150 \pm 180$ vation.

Bone fragments found at Haan $\left(53^{\circ} 8^{\prime} \mathrm{N}, 6^{\circ} 14^{\prime} \mathrm{E}\right)$ during salvage exca-

West Germany

UCLA-1949. Manching

$300 \pm 100$

Bone fragments, femur and tibia from Manching, Bavaria $\left(48^{\circ} 43^{\prime} \mathrm{N}\right.$, $\left.11^{\circ} 31^{\prime} \mathrm{E}\right)$. Comment (RP): question was if these bones belonged to late Roman settlement. Date confirms that they are recent intrusive.

\section{German Pleistocene Hominid series}

The following dates were determined to provide absolute time placement for several German fossils of uncertain age due to lack of direct dating. Subm by R Protsch.

UCLA-1783. Offnet Höhle

$$
13,100 \pm 70
$$

Bone from infant found in Offnet cave, Bavaria $\left(48^{\circ} 51^{\prime} \mathrm{N}, 10^{\circ} 31^{\prime} \mathrm{W}\right)$. From colln of G Glowatzki, Munich.

UCLA-1869. Neuessing

$18,200 \pm 200$

Tibia of anatomically modern man, Magdalenian culture, from Neuessing, Bavaria $\left(48^{\circ} 51^{\prime} \mathrm{N}, 10^{\circ} 31^{\prime} \mathrm{W}\right)$. From colln of G Glowatzki.

\section{UCLA-2360. Paderborn}

$25,650 \pm 1300$

Upper Pleistocene hominid from vicinity of Paderborn $\left(51^{\circ} 43^{\prime} \mathrm{N}, 8^{\circ}\right.$ $44^{\prime}$ E); date based on amino acids (Henke \& Protsch, 1978).

UCLA-2361. Kelsterbach

$\mathbf{2 9 , 0 0 0} \pm \mathbf{5 3 0}$

Upper Pleistocene hominid from Kelsterbach next to Frankfurt airport $\left(50^{\circ} 6^{\prime} \mathrm{N}, 8^{\circ} 41^{\prime} \mathrm{E}\right)$. Date based on amino acids extracted from calotte (Protsch, 1986).

UCLA-2363. Hanöfersand

Upper Pleistocene hominid from Hanöfersand near Hamburg $\left(53^{\circ} 33^{\prime}\right.$ $\mathrm{N}, 10^{\circ} \mathrm{E}$ ). From frontal bone (Bräuer, 1980).

General Comment (RP): dates provide first direct ${ }^{14} \mathrm{C}$ dates for these important Upper Pleistocene hominids which can be placed into evolutionary sequence. 
Africa

\section{Subsaharan Fossil Hominid series}

Fossil bone samples were coll in Africa to determine whether anatomically modern man appears later, is sympatric with or even antedates Neanderthals. Coll and subm by R Protsch.

\section{UCLA-1740. Olduvai Hominid I} $\left.13^{\prime} \mathrm{E}\right)$.

Postcranial fragments from Olduvai Gorge, Tanzania $\left(2^{\circ} 50^{\prime} \mathrm{N}, 35^{\circ}\right.$

UCLA-1741. Naivasha

$10,850 \pm 330$ $\left.26^{\prime} \mathrm{E}\right)$.

From living site of Naivasha, $80 \mathrm{~km} \mathrm{NE}$ of Nairobi, Kenya $\left(44^{\prime} \mathrm{S}, 36^{\circ}\right.$

\section{UCLA-1 742. Saldanha}

$40,680 \pm 3000$

Bone fragments from Saldanha hominid found at Elandsfontein farm $13 \mathrm{~km}$ WSW of Hopefield, South Africa $\left(33^{\circ} 5^{\prime} \mathrm{S}, 18^{\circ} 20^{\prime} \mathrm{E}\right)$. Same as GrN-6593: 40,570 $\begin{array}{r}+1250 \\ -1080 .\end{array}$

UCLA-1744. Fish Hoek

$35,630 \pm 2500$

Bone (Equus capensis) from Fish Hoek, $23 \mathrm{~km} \mathrm{~S}$ of Capetown, South Africa $\left(33^{\circ} 56^{\prime} \mathrm{S}, 18^{\circ} 28^{\prime} \mathrm{E}\right)$. For assoc charcoal dates, 36,000 \pm 2400 : UCLA-1235, see Bada et al (1974).

UCLA-1745B. Florisbad

$38,680 \pm 2000$

Bone (Hippopotamus amphibius) from Florisbad, South Africa (28 $46^{\prime}$ $\left.\mathrm{S}, 26^{\circ} 5^{\prime} \mathrm{E}\right)$. For assoc wood dates 38,250 \pm 3800 : UCLA-1 745C, see Bada et al (1974).

UCLA-1746A. Matjes River

$10,120 \pm 200$

From proto-Bushman level, C-1342, at Matjes R rock shelter near Knysna, South Africa $\left(34^{\circ} 4^{\prime} \mathrm{S}, 23^{\circ} 3^{\prime} \mathrm{E}\right)$.

UCLA-1746B. Matjes River.

$9230 \pm 160$

From Wilton People level, SSII/SSIII/SSIV hominid.

UCLA-1746D. Matjes River

$7380 \pm 120$

From pre-Bushman level, C-1271/C-1272 hominid.

UCLA-1747B. Lukenya Hill

$13,150 \pm 90$

Bones (Connochaetes taurinus) from Lukenya Hill, $60 \mathrm{~km}$ SE of Nairobi, Kenya $\left(1^{\circ} 17^{\prime} \mathrm{S}, 36^{\circ} 50^{\prime} \mathrm{E}\right)$.

UCLA-1747D. Lukenya Hill

$16,750 \pm 150$

Bone (Gazella thomsoni ?) and assoc charcoal found at loc 98.25-15. 
UCLA-1750C. Mumbwa

Hominid remains from Mumbwa 1 , Stratum V, Zambia $\left(15^{\circ} \mathrm{S}, 27^{\circ} 1^{\prime}\right.$ E). Relevant fauna-based collagen dates: Stratum V, 20,450 \pm 340 : UCLA$1750 \mathrm{~B}$ and Stratum IV, 18,000 \pm 370 : UCLA-1750B (Bada et al, 1974).

\section{UCLA-1754C. Border Cave}

$\mathbf{3 4 , 4 0 0} \pm \mathbf{2 5 0 0}$

Fragmentary bone material from Border Cave, Natal on W scarp of Lebombo Range $\left(27^{\circ} 8^{\prime} \mathrm{S}, 32^{\circ} \mathrm{E}\right)$ South Africa. From $76 \mathrm{~cm}$ depth in First White Ash layer.

UCLA-1754D. Border Cave

$34,800 \pm 2500$

Fragmentary bone from First White Ash layer at $100 \mathrm{~cm}$ depth.

UCLA-1 754E. Border Cave

45,000

Fragmentary bone from Second White Ash layer at $140 \mathrm{~cm}$ depth.

UCLA-1755B. Bushman Rock Shelter

$27,400 \pm 1600$

Bone fragments from Bushman Rock Shelter, Level 14, E Transvaal near Ohrigstad, South Africa ( $\left.24^{\circ} 49^{\prime} \mathrm{S}, 30^{\circ} 33^{\prime} \mathrm{E}\right)$.

UCLA-1755C. Bushman Rock Shelter

$31,900 \pm 1800$

Bone fragments from Level 18.

UCLA-1755D. Bushman Rock Shelter

$34,380 \pm 4300$

Bone fragments from level 21.

UCLA-1756. Gamble's Cave

$8210 \pm 260$

Bone from Gamble's Cave on Miti Mingi Farm, $11 \mathrm{~km}$ SE Elmenteita, Kenya $\left(26^{\circ} \mathrm{S}, 36^{\circ} 14^{\prime} \mathrm{E}\right)$.

\section{UCLA-1757. Bromhead's site}

$7410 \pm 160$

Bone fragments from Bromhead's site, $8 \mathrm{~km} \mathrm{~N}$ of Gamble's Cave on S shore of Lake Nakuru.

General Comment (RP): detailed implications of this fossil man study have been presented in Protsch (1973). Concordant charcoal, shell, collagen ${ }^{14} \mathrm{C}$ dates and amino acid racemization dates are discussed in Bada et al (1974).

\section{REFERENCES}

Bada, J L, Schroeder, R A, Protsch, R and Berger, R, 1974, Concordance of collagen-based radiocarbon and aspartic acid racemization ages: US Natl Acad Sci Proc, v 71, p 914917.

Berger, R, 1975, Advances and results in radiocarbon dating: Early Man in America: World Archaeology, v 7, p 174-184.

Berger, R, DeAtley, S, Protsch, R and Willey, G R, 1974, Radiocarbon chronology for Seibal, Guatemala: Nature, v 252, no. 5483, p 472-473.

Berger, R, Horney, A G and Libby, W F, 1964, Radiocarbon dating of bone and shell from their organic components: Science, v 144, p 999-1001.

Berger, R and Libby, W F, 1969, UCLA radiocarbon dates IX: Radiocarbon, v 11, no. 1,

p 194-209.
Berger, R and Protsch, R, 1975, Identification of skeletal material found in a Greek hydria: J Paul Getty Mus Jour, v 1, p 23-24.

Berger, R, Protsch, R, Reynolds, R, Rozaire, C and Sackett, J R, 1971, New radiocarbon dates 
based on bone collagen of California Palaeo-Indians, in Stross, F H, ed, The application of the physical sciences to archaeology: Contr Univ California Archaeol Research Facility, $\mathrm{v} 12, \mathrm{p} 43-49$

Bräuer, G, 1980, Morphologische Affinitäten des jung-Pleistozänen Stirnbeins aus dem Elbmündungsgebiet bei Hanöfersand: Zeitschr Morphol Anthropol, v 71, p 1-42.

Connor, M (ms), 1985, Population structure and biological variation in the Late Woodland of western central Illinois: PhD dissert, Univ Chicago.

Ericson, J E and Berger, R, 1974, Late Pleistocene American obsidian tools: Nature, v 249, p 824-825.

Hayes, W C, 1962, Chronology: Egypt-to the end of the twentieth dynasty, in Edwards, I E S, Gadd, G J and Hammond, N G L, eds, Cambridge Ancient History: Cambridge, Cambridge Univ Press, p 1-23.

Henke, W and Protsch, R, 1978, Die Paderborner Calvaria: ein diluvialer Homo sapiens: Anthropolo Anz, v 36, p 85-108.

Ho, T-Y, Marcus, L F and Berger, R, 1969, Radiocarbon dating of petroleum-impregnated bone from tar pits at Rancho La Brea, California: Science, v 164, p 1051-1052.

Kaufman, T S, (ms) 1980, Early prehistory of the Clear Lake area, Lake County, California: PhD dissert, Univ California, Los Angeles.

Longin, R, 1971, New method of collagen extraction for radiocarbon dating: Nature, v 230, p 241-242.

Meighan, C W and Haynes, C V, 1970, The Borax Lake site revisited: Science, v 167, p 1213 1221

Olson, E A and Broecker, W S, 1961, Lamont natural radiocarbon measurements VII: Radiocarbon, v 3, p 141-175.

Orr, P C, 1968, Prehistory of Santa Rosa Island: Santa Barbara Mus Nat Hist, Santa Barbara, California.

Perino, G, 1973, The Late Woodland component at the Schild sites, Greene County, Illinois, in Brown, J A, ed, Late Woodland site archaeology in Illinois, I: Illinois Archaeol Survey Bull no. 9, p 141-210.

Protsch, R, (ms) 1973, The dating of upper Pleistocene subsaharan fossil hominids and their place in human evolution: with morphological and archaeological implications: $\mathrm{PhD}$ dissert, Univ California, Los Angeles.

1986, Radiocarbon dating of bone, in Zimmerman, M R and Angels, J L, eds, Dating and age determination of biological material: London, Croom Helm, $\mathrm{p} 1-38$.

Struever, S, (ms) 1968, A re-examination of Hopewell in eastern North America: PhD dissert, Univ Chicago.

Taylor, R E, Payne, L A, Prior, C A, Slota, P J, Jr, Gillespie, R, Gowlett, J A J, Hedges, R E B, Jull, A J T, Zabel, T H, Donahue, D J and Berger, R, 1985, Major revisions in the Pleistocene age assignments for North American human skeletons by ${ }^{14} \mathrm{C}$ accelerator mass spectrometry: Am Antiquity, v 50, p 136-140. 